\title{
The effect of curing mode of a high-power LED unit on bond strengths of dual- cure resin cements to dentin and CAD/CAM resin blocks
}

\author{
Yuna KANAMORI ${ }^{1}$, Rena TAKAHASHI ${ }^{1}$, Toru NIKAIDO ${ }^{1,2}$, Elias P. BAMIDIS ${ }^{3}$, Michael F. BURROW ${ }^{4}$ \\ and Junji TAGAMI ${ }^{1}$ \\ ${ }^{1}$ Department of Cariology and Operative Dentistry, Graduate School of Medical and Dental Sciences, Tokyo Medical and Dental University (TMDU), \\ 1-5-45 Yushima, Bunkyo-ku, Tokyo 113-8549, Japan \\ ${ }^{2}$ Department of Operative Dentistry, Division of Oral Functional Science and Rehabilitation, School of Dentistry, Asahi University, 1851 Hozumi, \\ Mizuho, Gifu 501-0296, Japan \\ ${ }^{3}$ Department of Restorative Dentistry and Periodontology, University Hospital of Munich, Ludwig-Maximilians-University, Goethestr. 70,80336 \\ Munich, Germany \\ ${ }^{4}$ Faculty of Dentistry, University of Hong Kong, Pokfulam, Hong Kong SAR, China \\ Corresponding author, Rena TAKAHASHI; E-mail: renatakahashi@hotmail.com
}

The purpose of this study was to investigate the effects of curing mode with a high-power LED curing unit (VALO) in terms of microtensile bond strength ( $\mu$ TBS) to dentin and microhardness of two dual-cure resin cements. Panavia V5 (V5) and Rely X Ultimate (RXU) were polymerized using one of three curing modes with VALO or self-cure mode to bond a CAD/CAM resin block to a flat dentin surface. Specimens were sectioned and subjected to $\mu$ TBS test. Vickers hardness values of V5 and RXU were also measured. Two-way ANOVA indicated curing mode and resin cement affected $\mu$ TBS. For self-cure mode, V5 had significantly higher $\mu$ TBS than RXU $(p<0.05)$. Higher irradiance did not always provide higher $\mu$ TBS of dual-cure resin cement to dentin. One-way ANOVA indicated the curing modes affected microhardness of each cement. As for microhardness of RXU, there were significant differences between selfcure mode and light cure modes $(p<0.05)$.

Keywords: Dual-cure resin cement, Microtensile bond strength, CAD/CAM, Irradiance, Microhardness

\section{INTRODUCTION}

Computer-aided design/computer aided manufacturing (CAD/CAM) technology has brought about great changes especially in the fields of prosthodontics and restorative dentistry ${ }^{1}$. Indirect dental restorations generated by $\mathrm{CAD} / \mathrm{CAM}$ technology have enabled the reduction in labor of dental laboratory technicians and enhanced quality control ${ }^{2}$. Various materials such as zirconia, lithium disilicate glass ceramics, leucite-reinforced glass ceramics, feldspathic ceramics, and resin composites are available for manufacturing as CAD/CAM blocks. CAD/ CAM resin blocks offer some advantages related to their machinability ${ }^{3)}$, allow simple intraoral repair), and cause less wear of opposing teeth ${ }^{5}$. However, the CAD/ CAM resin block is inferior to ceramics with respect to mechanical properties such as fracture resistance and wear.

Dual-cure resin cements are ideal luting agents to cement CAD/CAM dental prostheses to prepared teeth because of their superior retentive and mechanical properties $^{6)}$, better wear resistance ${ }^{7,8)}$, and reduced solubility ${ }^{9}$. In order to attain the greatest polymerization of dual-cure resin cements, the light reaching the cement beneath the restoration is one of the critical factors to achieve optimal properties of dual-cure resin cements. Previous studies have revealed that reduced irradiance could negatively influence the physicochemical properties of dual-cure resin cements ${ }^{10,11)}$. Currently, the third-generation of light-emitting diode (LED) curing units with multiple diodes (violet/blue diodes, polywave) are on the market (VALO, Ultradent, South Jordan, UT, USA). This unit is claimed by the manufacturer to be reaching a radiant exitance of $1,000 \mathrm{~mW} / \mathrm{cm}^{2}$ in Standard-power mode (Standard mode), 1,400 $\mathrm{mW} / \mathrm{cm}^{2}$ in High-power mode (High mode), and $3,200 \mathrm{~mW} / \mathrm{cm}^{2}$ in Xtra-power mode (Xtra mode).

Good bonding is essential to ensure the longevity of the tooth-restoration complex ${ }^{12)}$. Microtensile bond strength ( $\mu$ TBS) tests are widely used to assess the quality and degree of adhesion of resin cements ${ }^{13,14}$. Microhardness is regarded as a useful indirect indication to investigate the degree of polymerization of resinbased materials ${ }^{15,16)}$, though an absolute hardness value is not able to reveal the actual conversion of different materials ${ }^{17}$.

Thus, the purpose of this study was to investigate the effects of curing mode using a high-power LED curing unit in terms of $\mu \mathrm{TBS}$ and microhardness of two dual-cure resin cements. The null hypotheses tested are as follows: (i) the curing mode irradiance does not affect the bond strength of resin cement to dentin, or microhardness of resin cement, and (ii) the choice of dual-cure resin cement does not affect the bond strength of the cement to dentin regardless of the curing mode.

Color figures can be viewed in the online issue, which is available at J-STAGE.

Received Oct 22, 2018: Accepted Jan 2, 2019

doi:10.4012/dmj.2018-344 JOI JST.JSTAGE/dmj/2018-344 


\section{MATERIALS AND METHODS}

Detailed information about the materials used in this study is provided in Table 1.

\section{Dual-cure resin cements used in this study}

Two dual-cure resin cements, Panavia V5 (V5) with Panavia V5 Tooth Primer (Tooth Primer) (Kuraray Noritake Dental, Tokyo, Japan) and Rely X Ultimate (RXU) with Scotchbond Universal Adhesive (SBU; 3M ESPE, St. Paul, MN, USA) were used. Each cement was dispensed from an auto-mixing syringe delivered by the manufacturer.

\section{Preparation of $C A D / C A M$ resin slabs}

Five CAD/CAM resin blocks (KATANA AVENCIA Block, Kuraray Noritake Dental) were sectioned with a low-speed diamond saw (Isomet, Buehler, Lake Bluff, IL, USA) to obtain forty slabs with a thickness of $1 \mathrm{~mm}$. The surfaces of the slabs were finished with 600-grit silicon carbide paper under running water, and then airabraded using $50 \mu \mathrm{m}$ aluminum powder (Heraeus Kulzer Japan, Tokyo, Japan) at $0.1 \mathrm{MPa}$ with the air-abrasion tip perpendicularly positioned at $10 \mathrm{~mm}$ to the specimen surface for $10 \mathrm{~s}$, then cleaned ultrasonically for $3 \mathrm{~min}$ in distilled water. Each sectioned slab was measured with a digital caliper (Mitutoyo CD-15C, Mitutoyo, Kanagawa, Japan) to ensure a final thickness of $1.00 \pm 0.05 \mathrm{~mm}$.

\section{Measurement of irradiance and spectra}

A LED curing unit (VALO) was used in this study. The VALO light curing unit offers three types of curing modes: Standard mode, High mode, and Xtra mode. The VALO light curing unit was placed in a box to exclude light and the center of the aperture was fixed directly on a spectroradiometer (USR-45DA, Ushio, Tokyo, Japan). The light-receiving part of the spectroradiometer was 6 $\mathrm{mm}$ in diameter whilst the aperture of the VALO light curing unit tip was $9.6 \mathrm{~mm}$ in diameter. The radiant exitance and the wavelength of the three curing modes of the VALO light curing unit were measured using the spectroradiometer $\left(\mathrm{mW} / \mathrm{cm}^{2}\right)(0-\mathrm{mm})$. The irradiance of the three modes was also determined by keeping a 1-mm distance between the light curing unit tip and the light-receiving part of the radiometer (1-mm air). Furthermore, the irradiance of the three modes was determined through a 1-mm thick CAD/CAM slab by placing it on the aperture of the spectroradiometer (1-mm slab). The radiant exitance of each condition was measured five times respectively.

\section{Specimen preparation for the $\mu T B S$ test}

The present study's protocol was designed according to the guidelines of the Ethics Committee of the Graduate School and Hospital, Tokyo Medical and Dental University (No. D2013-022). Forty extracted intact human molar teeth were used. A flat superficial dentin tooth surface was exposed and subsequently finished with 600-grit silicon carbide paper under running water to create a standardized surface.
Prepared CAD/CAM resin slabs were treated with $37 \%$ phosphoric acid (K-etchant, Kuraray Noritake Dental) for $5 \mathrm{~s}$, rinsed with water, and air-dried. The slabs were randomly divided into two groups for the selected resin cements used, namely V5 or RXU. In the V5 group, a silane coupling agent (Clearfil Ceramic Primer Plus, Kuraray Noritake Dental) was applied to the surface of the slabs and gently air-dried. In the RXU group, SBU was applied to the surface of the slabs and gently air-thinned. Depending on the resin cement, either Tooth Primer or SBU was applied to the dentin surface. Each cement was used according to the manufacturers' instructions (see Table 1).

The first $1 \mathrm{~cm}$ of the mixed cement was dispensed and then discarded in order to ensure evenly mixed cement. The mixed cement paste of either V5 or RXU was placed on the surface of each CAD/CAM resin slab. According to curing mode, the teeth were randomly selected into four test groups for each resin cement: self-cure (Self-cure) mode, Standard mode, High mode, and Xtra mode. For Self-cure mode, the slab was luted to the dentin surface with one of the resin cements. A load of $0.5 \mathrm{~kg}$ was then used to eliminate excess cement, which was removed with a cotton pellet, and the specimen was kept in the dark for $30 \mathrm{~min}$ to ensure only self-cure activation. For Standard mode, High mode, and Xtra mode, after the slab was luted to the surface of the dentin, a load of 0.5 $\mathrm{kg}$ was used to eliminate excess resin cement, which was again removed with a cotton pellet. After removal of the seating force the specimens were light-cured using the VALO light curing unit for $20 \mathrm{~s}$ using one of the three curing modes, namely: $20 \mathrm{~s} \times 1$ in Standard mode; $4 \mathrm{~s} \times 5$ in High mode; and $3 \mathrm{~s} \times 6+2 \mathrm{~s} \times 1$ in Extra mode, through the resin slab.

\section{$\mu T B S$ test}

The bonded specimens were kept in water at $37^{\circ} \mathrm{C}$ for $24 \mathrm{~h}$. The top surface of the CAD/CAM resin slab was treated with $37 \%$ phosphoric acid (K-etchant) for 5 $\mathrm{s}$, rinsed with water and air-dried. A silane coupling agent (Clearfil Ceramic Primer Plus, Kuraray Noritake Dental) was applied to the surface and gently air-dried. A bonding agent (Clearfil SE Bond, Kuraray Noritake Dental) was applied to the surface, gently air-dried, and light cured for $10 \mathrm{~s}$. Then, a direct resin composite (Clearfil AP-X Shade A2, Kuraray Noritake Dental) was incrementally built up to a thickness approximately $2 \mathrm{~mm}$ high for the $\mu$ TBS test. Each tooth was cross-sectioned longitudinally with a low-speed diamond saw (Isomet) to produce beam-shaped specimens with an approximate surface area of $1 \times 1 \mathrm{~mm}^{2}$ at the bonded interface. Before the $\mu$ TBS test, the dimensions of each beam were determined with a digital caliper (Mitutoyo CD-15C). After this, each specimen was secured to a customized microtensile jig with a cyanoacrylate glue (Model Repair II Blue, Dentsply-Sankin, Tokyo, Japan) and placed in the testing apparatus (EZ-SX, Shimadzu, Kyoto, Japan) for the $\mu$ TBS test at a $1 \mathrm{~mm} / \mathrm{min}$ crosshead speed. 
Table 1 Materials used in this study

\begin{tabular}{|c|c|c|c|c|c|}
\hline Material & Code & Manufacturer & Compositions & Application instructions & Batch No. \\
\hline $\begin{array}{l}\text { Panavia V5 } \\
\text { Tooth primer }\end{array}$ & $\begin{array}{l}\text { Tooth } \\
\text { Primer }\end{array}$ & $\begin{array}{l}\text { Kuraray } \\
\text { Noritake } \\
\text { Dental, } \\
\text { Tokyo, Japan }\end{array}$ & $\begin{array}{l}\text { 10-MDP, original multifunctional } \\
\text { monomer, new polymerization } \\
\text { accelerator, HEMA, water, stabilizer } \\
\text { pH: } 2.0\end{array}$ & $\begin{array}{l}\text { Applied and rubbed on the } \\
\text { surface for } 20 \mathrm{~s} \text { and gently } \\
\text { air-dried. }\end{array}$ & 2U0035 \\
\hline
\end{tabular}

Bis-GMA, TEGDMA, aromatic multifunctional monomer, aliphatic multifunctional monomer, new chemical The paste from auto-mix

$\begin{array}{lll}\text { Panavia V5 } & & \begin{array}{l}\text { Kuraray } \\ \text { (Universal }\end{array} \text { V5 } \\ \text { shade) } & & \begin{array}{l}\text { Noritake } \\ \text { Dental }\end{array}\end{array}$

Scotchbond

Universal

SBU

3M ESPE,

Adhesive

Rely X

Ultimate

RXU

3M ESPE

(A1 shade) polymerization accelerator,

dl-camphorquinone,

photopolymerization accelerator, surface treated barium glass,

fluoroaluminosilicate glass,

fine particulate filler

10-MDP, dimethacrylate resins, HEMA, Vitrebond ${ }^{\mathrm{TM}}$ copolymer, filler, ethanol, water, initiators, silane pH: 2.7 syringe was placed, and light-cured for $20 \mathrm{~s}$ or left for $30 \mathrm{~min}$ in a dark place.

To dentin:

Applied and rubbed on the surface for $20 \mathrm{~s}$ and gently air-thinned for about $5 \mathrm{~s}$ until it does not move. To CAD/CAM resin block: Applied and rubbed on the surface for $20 \mathrm{~s}$ and gently air-thinned for about $5 \mathrm{~s}$ until it does not move.

Base:

silane treated glass powder, 2-propenoic acid, 2-methyl-, 1,1'-[1-(hydroxymethyl)1,2-ethanediyl] ester, reaction products with 2-hydroxy-1,3-propanediyl dimethacrylate and phosphorus oxide, TEGDMA, silane treated silica, oxide glass chemicals, sodium persulfate, tertbutyl peroxy-3,5,5-trimethylhexanoate, copper (ii) acetate monohydrate. Catalyst:

silane treated glass powder, substituted dimethacrylate, 1-benzyl5-phenylbarbic-acid, calcium salt, 1,12-dodecane dimethycrylate, sodium p-toluenesulfinate, silane treated silica, 2-propenoic acid, 2-methyl-, [(3-methoxypropyl)imino]di-2,1-, ethanediyl ester, calcium hydroxide, titanium dioxide

\begin{tabular}{lll} 
KATANA & & \\
AVENCIA & & $\begin{array}{l}\text { Kuraray } \\
\text { Block } \\
\text { (Size:12, } \\
\text { Shade:A3 LT) }\end{array}$ \\
K-etchant & - & $\begin{array}{l}\text { Dental } \\
\text { GEL }\end{array}$ \\
Clearfil & - & $\begin{array}{l}\text { Kuraray } \\
\text { Noritake } \\
\text { Dental }\end{array}$ \\
Ceramic & & $\begin{array}{l}\text { Kuraray } \\
\text { Primer Plus }\end{array}$ \\
\hline
\end{tabular}

The paste from auto-mix syringe was placed, and light-cured for $20 \mathrm{~s}$ or left for $30 \mathrm{~min}$ in a dark place.
UDMA, TEGDMA, aluminum filler $(20 \mathrm{~nm})$, silica filler $(40 \mathrm{~nm})$, pigments filler: 62 wt\%

$35 \%$ phosphoric acid, water, colloidal silica, dye

3-trimethoxysilylpropyl methacrylate, 10-MDP, ethanol

000204

Applied for 5 s, flushed by water, and gently air-dried

BP0053

10-MDP: 10-methacryloyloxydecyl dihydrogen phosphate; HEMA: 2-hydroxyethyl methacrylate; Bis-GMA: bisphenol-Adiglycidyl methacrylate; TEGDMA: triethyleneglycol dimethacrylate; UDMA: urethane-dimethacrylate monomer-1,6-bis[methecryloyloxy-2-ethoxycarbonylamino] 


\section{Failure modes}

After the $\mu$ TBS test, the debonded specimens were placed in epoxy resin (EpoxiCure 2, Buehler). The resin-embedded specimens were polished with wet silicon carbide papers $(600,800,1000,1200,1500$, and 2000-grit) and diamond pastes $(6,3,1$, and $0.25 \mu \mathrm{m})$ sequentially. After finishing, the specimens were cleaned ultrasonically for $3 \mathrm{~min}$ in distilled water. Then, the specimens were dried for $24 \mathrm{~h}$, gold sputter-coated, and examined with a scanning electron microscope (SEM; JSM-5310LV, JEOL, Tokyo, Japan) at 50× and 500× magnification. The failure modes were classified into the following six categories as illustrated in Fig. 1: Type A: cohesive failure in the resin block; Type B: complete adhesive failure at the interface between block and resin cement; Type C: cohesive failure in resin cement; Type $\mathrm{D}$ : complete adhesive failure at the interface between resin cement and dentin; Type $\mathrm{E}$ : cohesive failure in dentin; Type F: mixed failure.

Specimens preparations for microhardness test Specimens for the microhardness test were prepared according to the following method. A total of one hundred and four disc-shaped specimens of the resin cements ( $n=13$ /group) were made in molds (internal diameter $8 \times 2$ $\mathrm{mm}$ thickness). Transparent plastic strips were located at the top and bottom surfaces to guarantee smooth surfaces. For the Self-cure mode group, the specimens were placed in the dark for $30 \mathrm{~min}$ to provoke chemical reactions in the resin cement by only self-cure activation. For Standard mode, High mode, and Xtra mode groups, light curing was conducted through a 1 -mm thick CAD/ CAM resin slab placed on top of a transparent plastic strip for $20 \mathrm{~s}$ using the VALO light curing unit either with Standard mode $(20 \mathrm{~s} \times 1)$, High mode $(4 \mathrm{~s} \times 5)$, or Xtra mode $(3 \mathrm{~s} \times 6+2 \mathrm{~s} \times 1)$. The specimens were detached

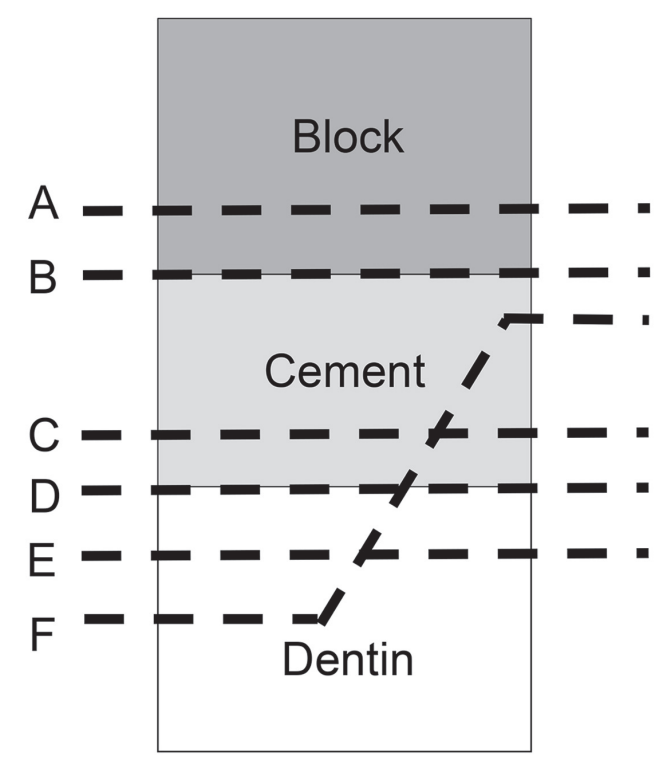

Fig. 1 Illustration of each failure mode. from the mold and stored in distilled water at $37^{\circ} \mathrm{C}$ for 24 h. Microhardness was then assessed using a Vickers hardness indenter (HM102, Mitutoyo) with a $50 \mathrm{~g}$ load for $15 \mathrm{~s}$. Measurements were made at three randomly chosen locations of the top surface of each specimen from the Standard mode, High mode, and Xtra mode groups. The three measurements per specimen were averaged, and thirteen microhardness mean values for each cement and curing mode were used for statistical analysis.

\section{Statistical analysis}

The Kolomogorov-Smirnov test indicated that the $\mu$ TBS and microhardness outcomes were normally distributed $(p>0.05)$. The $\mu$ TBS values were statistically analyzed by two-way ANOVA followed by the post-hoc Tukey's multiple comparison test. The microhardness data of each cement were separately analyzed by one-way ANOVA followed by the post-hoc Tukey's multiple comparison test. All the statistical procedures were performed at the 95\% confidence level using PASW Statistic 18 (IBM, Armonk, NY, USA).

\section{RESULTS}

\section{Measurement of the irradiance}

Spectra of the three output modes of the VALO light curing unit are shown in Fig. 2. Each mode indicated a distinctive smaller peak around $395 \mathrm{~nm}$ and a higher peak around 440 to $470 \mathrm{~nm}$. The results of the measured irradiance are summarized in Table 2. The radiant exitance of the light curing unit from the light guide tip ( 0 - $\mathrm{mm})$ with Standard mode, High mode, and Xtra mode were 954.6 $\pm 2.8,1,537.4 \pm 3.4$, and $2,734.0 \pm 20.1 \mathrm{~mW} / \mathrm{cm}^{2}$, respectively. The radiant exitance at a distance of $1-\mathrm{mm}$ without CAD/CAM resin slab (1-mm air) with Standard mode, High mode, and Xtra mode were $1,118.8 \pm 2.7$, $1,845.6 \pm 10.0,3,221.3 \pm 8.2 \mathrm{~mW} / \mathrm{cm}^{2}$, respectively, while the radiant exitance through the $1-\mathrm{mm}$ CAD/CAM resin slab (1-mm slab) with Standard mode, High mode, and Xtra mode were significantly reduced to $214.9 \pm 0.6$, $252.7 \pm 0.3$, and $440.5 \pm 1.5 \mathrm{~mW} / \mathrm{cm}^{2}$, respectively.

\section{$\mu T B S$ test}

The results of the $\mu$ TBS tests are shown in Table 3. Twoway ANOVA showed that two factors, "curing mode" and "resin cement", influenced dentin bond strength $(p<0.05)$. A significant interaction was identified between the two factors $(p<0.05)$. The results of $\mu$ TBS test for V5 were statistically higher than those of RXU for Self-cure and Standard modes $(p<0.05)$. On the other hand, there were no significant differences in the $\mu$ TBS results between V5 and RXU for High and Xtra modes $(p>0.05)$.

For V5, there were significant differences between the Self-cure and Standard modes $(p<0.05)$. However, there were no significant differences in $\mu$ TBS between the Self-cure and High modes, Self-cure and Xtra modes, Standard and High modes, Standard and Xtra modes, and High and Xtra modes ( $p>0.05)$.

For RXU, there were significant differences between the Self-cure and Standard modes, Self-cure and High 


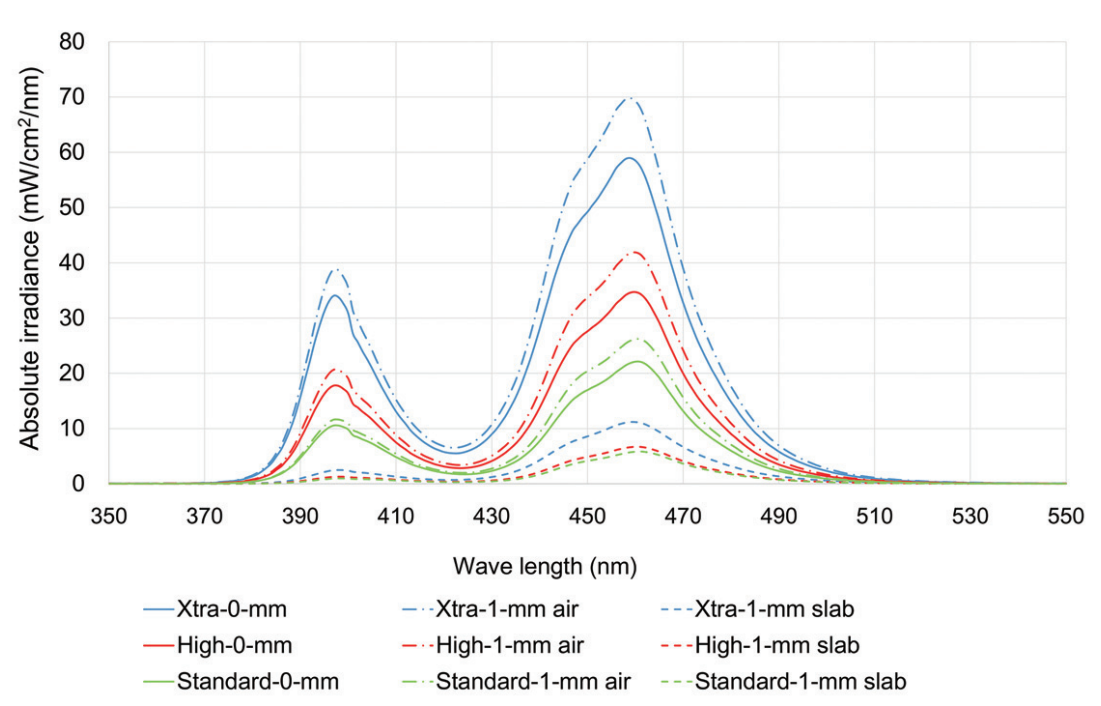

Fig. 2 Spectra without slabs at a distance of 0 and $1 \mathrm{~mm}$, and with $1 \mathrm{~mm}$ slabs in Standard, High and Xtra modes.

Table 2 Irradiance values of the light curing unit using different curing modes $\left(\mathrm{mW} / \mathrm{cm}^{2}\right)$

\begin{tabular}{lccc}
\hline & \multicolumn{3}{c}{ Curing mode } \\
\cline { 2 - 4 } & Standard mode & High mode & Xtra mode \\
\hline 0-mm & $954.6 \pm 2.8$ & $1,537.4 \pm 3.4$ & $2,734.0 \pm 20.1$ \\
1-mm air & $1,118.8 \pm 2.7$ & $1,845.6 \pm 10.0$ & $3,221.3 \pm 8.2$ \\
1-mm slab & $214.9 \pm 0.6$ & $252.7 \pm 0.3$ & $440.5 \pm 1.5$ \\
\hline
\end{tabular}

All values are the mean \pm SD.

Table 3 Mictotensile bond strengths of the resin cements to dentin using different curing modes (MPa)

\begin{tabular}{ccccc}
\hline & \multicolumn{3}{c}{ Curing mode } \\
\cline { 2 - 5 } & Self-cure mode & Standard mode & High mode & Xtra mode \\
\hline Panavia V5 & $36.7 \pm 7.1^{\mathrm{a}, \mathrm{b}}$ & $55.1 \pm 7.3^{\mathrm{c}, \mathrm{d}}$ & $49.2 \pm 7.3^{\mathrm{a}, \mathrm{c}, \mathrm{e}, \mathrm{A}}$ & $43.8 \pm 8.3^{\mathrm{b}, \mathrm{d}, \mathrm{e}, \mathrm{B}}$ \\
Rely X Ultimate & $19.6 \pm 7.4$ & $41.3 \pm 10.0^{\mathrm{f}, \mathrm{g}}$ & $54.6 \pm 12.7^{\mathrm{f}, \mathrm{h}, \mathrm{A}}$ & $44.0 \pm 13.6^{\mathrm{g}, \mathrm{h}, \mathrm{B}}$ \\
\hline
\end{tabular}

All values are the mean \pm SD.

Within the same row, the values with the same small superscript letters are not statistically different $(p>0.05)$.

Within the same column, the values with the same capital superscript letters are not statistically different $(p>0.05)$.

modes, and Self-cure and Xtra modes $(p<0.05)$. However, no significant differences in $\mu$ TBS between Standard and High modes, Standard and Xtra modes, and High and Xtra modes were observed $(p>0.05)$.

\section{Failure modes}

The failure mode results are summarized in Fig. 3. For the Self-cure mode, the predominant failure mode was a complete adhesive failure at the interface between resin cement and dentin (Type D) for both V5 and RXU. However, for all other modes, the percentage of Type D showed the tendency to decrease, and that of cohesive failure in dentin (Type E) increased for both V5 and RXU. In addition, the groups which had the highest percentage of Type E were Standard mode for V5, and High mode for RXU.

\section{Microhardness test}

The results of the microhardness tests are summarized in Table 4. For V5, there were significant differences between the Self-cure and Xtra modes, Standard and Xtra modes, and High and Xtra modes $(p<0.05)$. For 


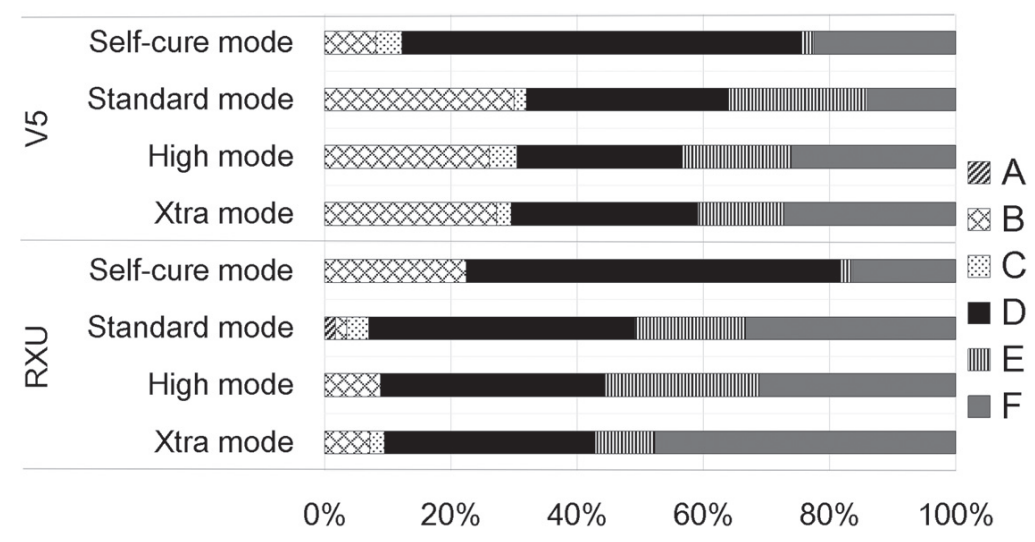

Fig. 3 The failure mode distribution.

Type A: cohesive failure in block; Type B: complete adhesive failure at the interface between block and resin cement; Type C: cohesive failure in resin cement; Type D: complete adhesive failure at the interface between resin cement and dentin; Type $\mathrm{E}$ : cohesive failure in dentin; Type F: mixed failure.

Table 4 Microhardness of the resin cements using different curing modes

\begin{tabular}{ccccc}
\hline & \multicolumn{3}{c}{ Curing mode } \\
\cline { 2 - 5 } & Self-cure mode & Standard mode & High mode & Xtra mode \\
\hline Panavia V5 & $23.6 \pm 3.5^{\mathrm{a}, \mathrm{b}}$ & $24.0 \pm 4.2^{\mathrm{a}, \mathrm{c}}$ & $25.4 \pm 1.8^{\mathrm{b}, \mathrm{c}}$ & $29.1 \pm 2.2$ \\
Rely X Ultimate & $28.5 \pm 5.7$ & $45.0 \pm 2.3^{\mathrm{d}, \mathrm{e}}$ & $44.0 \pm 2.0^{\mathrm{d}, \mathrm{f}}$ & $47.6 \pm 3.5^{\mathrm{e}, \mathrm{f}}$ \\
\hline
\end{tabular}

All values are the mean \pm SD.

Within the same resin cement, the values with small superscript letters are not statistically different $(p>0.05)$.

RXU, there were significant differences between the Self-cure and Standard modes, Self-cure and High modes, and Self-cure and Xtra modes $(p<0.05)$.

\section{DISCUSSION}

Multiple factors such as degree of conversion of resin cement, adhesion of dentin-resin cement and resin cement-CAD/CAM resin block interfaces are important to obtain optimal bond strength between the indirect restorative material and tooth structure ${ }^{15)}$. The curing mode and the choice of resin cement were the important factors influencing both dentin bond strengths of dualcure resin cements to $\mathrm{CAD} / \mathrm{CAM}$ resin blocks and microhardness of dual-cure resin cements. Therefore, both the first and the second null hypotheses were rejected based on the results.

The radiant exitance varied greatly under the different curing modes for the VALO light curing unit. The irradiance in 1-mm air showed a 17 to $20 \%$ increase compared to the irradiance of $0-\mathrm{mm}$ for each curing mode. This finding corresponds to the irradiance results observed using a MARC radiometer outlined in the study by Flury et $a l .{ }^{18)}$. In the present study, the lightreceiving part of the spectroradiometer was $6 \mathrm{~mm}$ in diameter and the aperture diameter of the light curing unit was $9.6 \mathrm{~mm}$. The size difference between the sensor of the spectroradiometer and the tip of a light-curing unit probably effects the results of the irradiance, which was collected by the sensor of the spectroradiometer. Thus, there was a possibility that higher irradiance was concentrated on the light-receiving part when the tip of the light-curing unit was kept at the distance of $1 \mathrm{~mm}$, compared to that of $0-\mathrm{mm}$ in the present study, or it could be that the light output distribution was not focused at the center of the light tip, which might have also influenced the results obtained ${ }^{19}$.

The irradiance measured through the 1-mm slab showed more than a $70 \%$ decrease in light output compared with 0 -mm for each curing mode. Not only does the restoration thickness reduce the light reaching the dual-cure resin cement beneath a restoration but also the opacity, filler particles, resin matrix composition and light curing unit also affect the irradiance ${ }^{20,21)}$. As with the resin material, other tooth colored restorative materials such as ceramics also cause a reduction in light transmission ${ }^{18)}$. Although the minimum thickness required for KATANA AVENCIA blocks is $1 \mathrm{~mm}$ at the axial wall, the thickness of CAD/CAM resin restorations will occasionally exceed $3 \mathrm{~mm}$ clinically. 
The VALO light curing unit delivered a similar emission spectrum across the three curing modes ${ }^{22)}$. The curing unit emitted light as three peaks that corresponded to the three types of LED chip used. The curing unit is comprised of four LEDs, one LED has a peak wavelength in light emission of $405 \mathrm{~nm}$ which appeared as a low and mild peak at around $400 \mathrm{~nm}$. Another LED has a peak wavelength in light emission of $445 \mathrm{~nm}$, while the other two LEDs have a peak wavelength in light emission of $465 \mathrm{~nm}$. As a result, the superimposition of these two peaks caused a small extension of the peak at the wavelength from 440 to $470 \mathrm{~nm}^{18,22)}$.

The light curing time was set at $20 \mathrm{~s}$ regardless of curing mode of the curing unit in the present study. The total energy output i.e. radiant energy $\left(\mathrm{J} / \mathrm{cm}^{2}\right)$, was calculated by multiplying the radiant exitance $(\mathrm{mW} /$ $\mathrm{cm}^{2}$ ) by the total exposure time in seconds. It has been reported that the total energy of the irradiation has a close relationship with the mechanical properties of photo-polymerized dental material ${ }^{23,24)}$. This principle was named the "total energy principle" or the "exposure reciprocity law". In contrast, several studies have reported that irradiance and light curing time separately affect the polymerization progress and subsequently the physical properties ${ }^{25-27)}$. Increasing the irradiance led to rapid polymerization, resulting in short chain lengths of the polymer, and reduced the propagation average per radical resulting in a compromise of the final degree of conversion ${ }^{28,29)}$. Additionally, lower irradiance will cause fewer free radicals, which results in their delayed diffusion thus limiting the ability to achieve higher degrees of conversion. This favors chain propagation over termination and extends the lifetime of the radical. Furthermore, the temperature increase caused by light activation will influence the degree of conversion ${ }^{28}$. The source of heat is from the light curing unit itself as well as the generation of heat from the polymerization reaction. Although the temperature change was not measured, the higher energy produced during the Xtra mode would have increased the heat generated from the light source. These findings are the probable reasons for the results that higher irradiance and total energy did not always improve dentin bond strengths nor microhardness of dual-cure resin cements and occasionally caused a deterioration in the present study.

For a single material, the rate of monomer conversion correlates with its microhardness ${ }^{30)}$. The microhardness of V5 in Self-cure mode was similar to the Standard mode and High mode. Meanwhile, microhardness of RXU in Self-cure mode was significantly lower than that of the other modes. Under clinical conditions, it seems likely that light will be incapable of reaching parts of a cavity during cementation of a restoration. Regarding the performance of dual-cure resin cement, RXU was influenced more by a change in the radiant exposure and curing mode than V5.

The specimen configuration has both dentin-resin cement and resin cement-CAD/CAM resin block adhesive interfaces. The self-curing interaction between the Tooth Primer or SBU, which includes co-initiators, and the resin cement, which includes an initiator, may improve polymerization activities while they come into contact but in the absence of light exposure ${ }^{13)}$. For V5, dentin was treated with Tooth Primer and CAD/CAM resin block was treated with a separate silane primer. For RXU, SBU was used for pre-treatment of both the dentin and CAD/CAM resin block. SBU is a so-called 'universal' adhesive which also contains a silane coupling agent that is claimed to be effective for bonding to both tooth and restoration surfaces. However, SBU contains many other ingredients such as 10-MDP, silane and water into one solution which may reduce the activity of functional monomers (10-MDP, silane) which therefore reduces the efficiency of adhesion to the substrates. The reduced effect of the silane in SBU was previously reported ${ }^{31}$. This is due to the relatively low $\mathrm{pH}$ (2.7) of the SBU solution that promotes dehydration condensation of the silane. It was claimed that the bond strength of SBU to $\mathrm{CAD} /$ CAM resin block would significantly drop after artificial ageing compared to 24 -h storage $^{31}$. In the present study, only 24-h bond strengths were investigated, thus the resin cement-CAD/CAM resin block interface was not so weak when analyzing the failure mode.

On the dentin, the adhesive was independently light cured before the luting of the indirect restoration which resulted in a higher bond strength than simultaneous colight-curing of the adhesive and resin cement ${ }^{32-34}$. Light curing the adhesive separately improves the adhesive interface and thus stabilizes the dentin hybrid layer. In the present study, SBU applied to dentin was light cured simultaneously with the resin cement. If SBU was light cured separately, higher bond strengths might have been obtained. Further studies such as evaluating the long-term durability and different curing strategies for SBU should be undertaken in order to achieve greater reliability for clinical applications.

\section{CONCLUSION}

Curing mode affected both dentin bond strengths of dual-cure resin cements to $\mathrm{CAD} / \mathrm{CAM}$ resin blocks, and microhardness of dual-cure resin cements. Higher irradiance did not always provide higher bond strength of the dual-cure resin cement to dentin. The degree of conversion did not always correlate with the dentin bond strengths.

\section{ACKNOWLEDGMENTS}

The authors are thankful to NIKKISO Co, Ltd Hakusan Factory for its assistance to measure the light irradiance and the wavelength of the LED light curing unit, and Kuraray Noritake Dental and 3M ESPE for their material donation. This study was supported by a Grantin-Aid for Scientific Research (No. 17K17122) from the Ministry of Education, Science, Sports, Culture, and Technology, Japan. 


\section{REFERENCES}

1) Alghazzawi TF. Advancements in CAD/CAM technology: Options for practical implementation. J Prosthodont Res 2016; 60: 72-84.

2) Miyazaki T, Hotta Y, Kunii J, Kuriyama S, Tamaki Y. A review of dental CAD/CAM: current status and future perspectives from 20 years of experience. Dent Mater J 2009; 28: 44-56.

3) Ruse ND, Sadoun MJ. Resin-composite blocks for dental CAD/CAM applications. J Dent Res 2014; 93: 1232-1234.

4) Rocca GT, Bonnafous F, Rizcalla N, Krejci I. A technique to improve the esthetic aspects of $\mathrm{CAD} / \mathrm{CAM}$ composite resin restorations. J Prosthet Dent 2010; 104: 273-275.

5) Zhi L, Bortolotto T, Krejci I. Comparative in vitro wear resistance of CAD/CAM composite resin and ceramic materials. J Prosthet Dent 2016; 115: 199-202.

6) Nakamura T, Wakabayashi K, Kinuta S, Nishida H, Miyamae M, Yatani H. Mechanical properties of new self-adhesive resin-based cement. J Prosthodont Res 2010; 54: 59-64.

7) Furuichi T, Takamizawa T, Tsujimoto A, Miyazaki M, Barkmeier WW, Latta MA. Mechanical properties and slidingimpact wear resistance of self-adhesive resin cements. Oper Dent 2016; 41: E83-92.

8) Takamizawa T, Barkmeier WW, Latta MA, Berry TP, Tsujimoto A, Miyazaki M. Simulated wear of self-adhesive resin cements. Oper Dent 2016; 41: 327-338.

9) Hill EE, Lott J. A clinically focused discussion of luting materials. Aust Dent J 2011; 56 Suppl 1: 67-76.

10) Ilie N, Hickel R. Correlation between ceramics translucency and polymerization efficiency through ceramics. Dent Mater 2008; 24: 908-914.

11) Kilinc E, Antonson SA, Hardigan PC, Kesercioglu A. The effect of ceramic restoration shade and thickness on the polymerization of light- and dual-cure resin cements. Oper Dent 2011; 36: 661-669.

12) Heintze SD. Clinical relevance of tests on bond strength, microleakage and marginal adaptation. Dent Mater 2013; 29: 59-84.

13) Tagami A, Takahashi R, Nikaido T, Tagami J. The effect of curing conditions on the dentin bond strength of two dualcure resin cements. J Prosthodont Res 2017; 61: 412-418.

14) Kawaguchi-Uemura A, Mine A, Matsumoto M, Tajiri $Y$, Higashi M, Kabetani T, et al. Adhesion procedure for CAD/ CAM resin crown bonding: Reduction of bond strengths due to artificial saliva contamination. J Prosthodont Res 2018; 62 : 177-183.

15) Bouschlicher MR, Rueggeberg FA, Wilson BM. Correlation of bottom-to-top surface microhardness and conversion ratios for a variety of resin composite compositions. Oper Dent 2004; 29: 698-704.

16) Gregor L, Bouillaguet S, Onisor I, Ardu S, Krejci I, Rocca GT. Microhardness of light- and dual-polymerizable luting resins polymerized through 7.5-mm-thick endocrowns. J Prosthet Dent 2014; 112: 942-948.

17) Poggio C, Lombardini M, Gaviati S, Chiesa M. Evaluation of Vickers hardness and depth of cure of six composite resins photo-activated with different polymerization modes. J Prosthet Dent 2012; 15: 237-241.
18) Flury S, Lussi A, Hickel R, Ilie N. Light curing through glass ceramics with a second- and a third-generation LED curing unit: effect of curing mode on the degree of conversion of dual-curing resin cements. Clin Oral Investig 2013; 17: 21272137.

19) Price RB, Ferracane JL, Shortall AC. Light-curing units: a review of what we need to know. J Dent Res 2015; 94: 11791186.

20) Musanje L, Darvell BW. Curing-light attenuation in filledresin restorative materials. Dent Mater 2006; 22: 804-817.

21) Lise DP, Van Ende A, De Munck J, Yoshihara K, Nagaoka N, Cardoso Vieira LC, et al. Light irradiance through novel CADCAM block materials and degree of conversion of composite cements. Dent Mater 2018; 34: 296-305.

22) Harlow JE, Sullivan B, Shortall AC, Labrie D, Price RB. Characterizing the output settings of dental curing lights. J Dent 2016; 44: 20-26.

23) Price RB, Felix CA, Andreou P. Effects of resin composite composition and irradiation distance on the performance of curing lights. Biomaterials 2004; 25: 4465-4477.

24) Leprince JG, Hadis M, Shortall AC, Ferracane JL, Devaux J, Leloup G, et al. Photoinitiator type and applicability of exposure reciprocity law in filled and unfilled photoactive resins. Dent Mater 2011; 27: 157-164.

25) Musanje L, Darvell BW. Polymerization of resin composite restorative materials: exposure reciprocity. Dent Mater 2003; 19: 531-541.

26) Asmussen E, Peutzfeldt A. Polymerization contraction of resin composite vs. energy and power density of light-cure. Eur J Oral Sci 2005; 113: 417-421.

27) Peutzfeldt A, Asmussen E. Resin composite properties and energy density of light cure. J Dent Res 2005; 84: 659-662.

28) FengL,SuhBI.Exposurereciprocitylawinphotopolymerization of multi-functional acrylates and methacrylates. Macromol Chem Phys 2007; 208: 295-306.

29) Faria ESAL, Pfeifer CS. Effectiveness of high-power LEDs to polymerize resin cements through ceramics: An in vitro study. J Prosthet Dent 2017; 118: 631-636.

30) Ferracane JL. Correlation between hardness and degree of conversion during the setting reaction of unfilled dental restorative resins. Dent Mater 1985; 1: 11-14.

31) Yoshihara K, Nagaoka N, Sonoda A, Maruo Y, Makita Y, Okihara T, et al. Effectiveness and stability of silane coupling agent incorporated in 'universal' adhesives. Dent Mater 2016; 32: 1218-1225.

32) Rathke A, Hokenmaier G, Muche R, Haller B. Effectiveness of the bond established between ceramic inlays and dentin using different luting protocols. J Adhes Dent 2012; 14: 147154 .

33) Luhrs AK, Pongprueksa P, De Munck J, Geurtsen W, Van Meerbeek B. Curing mode affects bond strength of adhesively luted composite $\mathrm{CAD} / \mathrm{CAM}$ restorations to dentin. Dent Mater 2014; 30: 281-291.

34) Araoka D, Hosaka K, Nakajima M, Foxton R, Thanatvarakorn $\mathrm{O}$, Prasansuttiporn $\mathrm{T}$, et al. The strategies used for curing universal adhesives affect the micro-bond strength of resin cement used to lute indirect resin composites to human dentin. Dent Mater J 2018; 37: 506-514. 